DOE-ER-40757-086

UTEXAS-HEP-96-17

\title{
NEW COLLIDER BOUND ON LIGHT GRAVITINO MASS
}

\author{
Duane A. Dicus \\ Center for Particle Physics \\ The University of Texas at Austin \\ Austin, Texas 78712 \\ S. Nandi \\ Department of Physics \\ Oklahoma State University \\ Stillwater, OK 74078 \\ and \\ Center for Particle Physics \\ The University of Texas at Austin \\ Austin, Texas 78712
}

\begin{abstract}
In supergravity theories with a very light gravitino, the gluino decays dominantly to a gluon and a gravitino. This results in a much larger missing $E_{T}$ for the multijet final states in hadronic colliders. We use the latest Tevatron data for the multijet final states to set a new absolute lower bound of $3.0 \times 10^{-13} \mathrm{GeV}$ for the light gravitino mass.
\end{abstract}

\section{Introduction}

Recent observation of $e^{+} e^{-} \gamma \gamma+\mathbb{E}_{T}$ events [1] by the CDF collaboration has generated a great deal of interest in the supergravity theories with a light gravitino [2]. In this scenario, the produced selectron decays to electron and the lightest neutralino which then decays to a photon and a light gravitino [3]. This gravitino can be very very light or superlight without affecting the above interpretation [3]. [Many other consequences of this interpretation are also being pursued [4].] However, if the gravitino $\tilde{G}$, is extremely light with $m_{\tilde{G}}$ in the range

\footnotetext{
*On sabbatical leave at the University of Texas at Austin.
} 
of $10^{-14}$ to $10^{-12} \mathrm{GeV}$, its gravitational interactions becomes comparable to the Standard Model $(S M)$ gauge interactions. For example, for such a very light gravitino, the gluino decays dominantly to a gluon plus gravitino, instead of the usual minimal supergravity mode, $q \bar{q} \tilde{Z}_{1}$. As a result, we get a much higher $\not_{T}$ for the ensuing multijet events. In addition such non-minimal $S G$ theories have a very light (essentially massless) scalar, $S$ and pseudoscalar, $P$ particle. These can be produced in association with a gluon (as $g S$ or $g P)$ in the $g g$ subprocess in the hadronic colliders. The cross sections for such productions are very large, since the coupling is proportional to $\kappa\left(m_{\tilde{g}} / m_{\tilde{G}}\right)$ and there is no phase space suppression. The net result of such theories is that we get multijet events with much larger $p_{T}$ than in the usual minimal supergravity theories. The CDF collaboration has set an upper limit, $\sigma<1.4 p b$ for the multijet cross section having $\mathbb{E}_{T}>50 \mathrm{GeV}$, and satisfying other cuts [5]. In this work, we use this result to set a lower bound of $3.0 \times 10^{-13} \mathrm{GeV}$ for the superlight gravitino mass. This is a substantial improvement over the previous lower bound of $2 \times 10^{-14} \mathrm{GeV}$ [6].

\section{Superlight or Very Light Gravitino}

In this section, we briefly recall the main features of the Non-Minimal Supergravity Theories (NMSG) with a superlight or very light gravitino. The interaction of a gravitino is, of course, purely gravitational. However, for a superlight or a very light gravitino, this interaction can be important in the laboratory. This is because a very light gravitino, $\psi_{\mu}$ behaves like a goldstino, $\chi$ with the replacement $\psi_{\mu}=i \sqrt{2 / 3} m_{\tilde{G}}^{-1} \partial_{\mu} \chi$. Its effective gravitational coupling becomes $\left(m_{\tilde{g}} / m_{\tilde{G}}\right) \kappa$ where $\kappa \equiv \sqrt{4 \pi G}$, where $G$ is Newton's-constant. This coupling becomes comparable to the gauge couplings of the $S M$, for a very very light gravitino, $\tilde{G}$ with a mass $m_{\tilde{G}} \sim 10^{-14} \mathrm{GeV}$. Is such a light gravitino allowed? For a general $S G$ theory, the gravitino mass depends on two arbitrary functions of the chiral superfields $(z), \mathcal{G}\left(z, z^{*}\right)$ and $f_{a b}(z) \cdot \mathcal{G}\left(z, z^{*}\right)$ multiplies the scalar kinetic term, and is called the Kähler potential while $f_{a b}(z)$ multiplies the gaugino kinetic term. For a minimal $S G$ theory, $\mathcal{G}\left(z, z^{*}\right)$ is a polynomial, and $f_{a b}(z)=\delta_{a b}$. In this case, the gravitino mass is necessarily in the weak scale. However, for general choices of these functions, which is the non-minimal $S G$ theory, the gravitino mass is arbitrary. The possible choices are: $m_{\tilde{G}} \simeq m_{W}^{2} / M_{P L}$ (superlight gravitino), $m_{\tilde{G}} \simeq m_{W}$ (usual SG), $m_{\tilde{G}} \simeq M_{P L}$ (ultra-heavy gravitino), $m_{\tilde{G}} \simeq\left(m_{W} / M_{P L}\right)^{n} M_{P L}$ with $1<n<2$ (very light gravitino). In this phenomenological work we consider $m_{\tilde{G}}$ in the range $10^{-14}$ to $10^{-12} \mathrm{GeV} . m_{\tilde{G}} \sim 2 \times 10^{-14} \mathrm{GeV}$ is the previous lower bound established us, and for $m_{\tilde{G}}>10^{-10} \mathrm{GeV}$, the theory behaves like the usual minimal supergravity theory (UMSG). A gravitino in the above mass range is necessarily the lightest supersymmetric particle (LSP). This gives rise to new decay modes for SUSY particles not present in the UMSG, and thus significantly alters the collider events topology. 


\section{Collider Productions and Decays}

In this section, we consider the productions of gluinos, gravitinos and scalar $S$ and pseudoscalar $P$ particles in hadronic colliders such as Tevatron and LHC. $(S$ and $P$ are the essentially massless particles left over from the hidden section after the super-Higgs mechanism.) We include the usual supersymmetric gauge interactions as well as the gravitational interactions involving the superlight gravitinos. The processes we consider are

$$
\begin{aligned}
& \bar{p} p \rightarrow \tilde{g} \tilde{g} \\
& \bar{p} p \rightarrow \tilde{g} \tilde{G} \\
& \bar{p} p \rightarrow g S+g P
\end{aligned}
$$

where $g$ stands for the gluon. The relevant interactions are [7]

$$
\begin{aligned}
e^{-1} \mathcal{L} & =\frac{1}{4} \kappa \bar{\lambda}^{a} \gamma^{\rho} \sigma^{\mu \nu} \psi_{\rho} F_{\mu \nu}^{a}+\frac{i}{2} \bar{\lambda}^{a} \not D \lambda^{a} \\
& +\frac{1}{4} \kappa \alpha S\left(F_{\mu \nu}^{a} F^{\mu \nu a}+\bar{\lambda}^{a} \not D \lambda^{a}\right) \\
& +\frac{1}{8} \kappa \alpha P\left[F_{\mu \nu}^{a} F^{\mu \nu a}-\frac{1}{2} e^{-1} D_{\mu}\left(e \bar{\lambda}^{a} \gamma_{5} \gamma^{\mu} \lambda^{a}\right)\right] \\
& +\beta S \bar{\lambda}^{a} \lambda^{a}+\text { usual super QCD terms. }
\end{aligned}
$$

The couplings $\alpha$ and $\beta$ are somewhat model dependent in the sense that their values depend on the specific choices of the functions $\mathcal{G}\left(z, z^{*}\right)$ and $f_{a b}(z)$. For a wide class of models with $f_{a b}(z)=\delta_{a b} f(z)$ and $\mathcal{G}\left(z, z^{*}\right)=-3 \ln \kappa\left(z+z^{*}\right)$, we get

$$
\alpha=-\sqrt{2 / 3} m_{\tilde{g}} / m_{\tilde{G}}, \quad \beta \sim 0\left(\kappa m_{\tilde{g}}\right) .
$$

First we consider the production processes (1a)-(1c) with the interaction given by eqs. (2) and (3). At the Tevatron and the LHC, we find the gluon-gluon fusion to be the dominant subprocess. For the process (1a), in addition to the usual SUSY QCD diagrams, we have the additional diagrams due to the t- and u-channel gravitino exchanges. For the gluon-gluon subprocess, the cross section is obtained to be 


$$
\sigma(g g \rightarrow \tilde{g} \tilde{g})=\frac{\left(1-\frac{4 m_{\tilde{g}}^{2}}{S}\right)^{\frac{1}{2}}}{256 \pi s} \int_{-1}^{1} d z \sum\left|M_{\text {Total }}\right|^{2}
$$

where the summation is over both spin and color and

$$
\begin{aligned}
\sum\left|M_{\text {Total }}\right|^{2} & =\left(\frac{\kappa^{2}}{6} \frac{m_{\tilde{g}}^{2}}{m_{\tilde{G}}^{2}}\right)^{2}\left(t u-s m_{\tilde{g}}^{2}\right)\left[\frac{8 t^{2}}{\left(t+m_{\tilde{g}}^{2}\right)^{2}}+\frac{8 u^{2}}{\left(u+m_{\tilde{g}}^{2}\right)^{2}}+\frac{2 s m_{\tilde{g}}^{2}}{\left(t+m_{\tilde{g}}^{2}\right)\left(u+m_{\tilde{g}}^{2}\right)}\right] \\
& -\left(\kappa^{2} \frac{m_{\tilde{g}}^{2}}{m_{\tilde{G}}^{2}}\right)\left(4 \pi \alpha_{s}\right)\left[\frac{1}{u} \frac{m_{\tilde{g}}^{2} t^{2}+m_{\tilde{g}}^{4} s-t^{2} u}{\left(t+m_{\tilde{g}}^{2}\right)}\right. \\
& +\frac{1}{s} \frac{s t^{2}-t^{3}-m_{\tilde{g}}^{2} s t}{\left(t+m_{\tilde{g}}^{2}\right)}+\frac{1}{t} \frac{m_{\tilde{g}}^{2} u^{2}+m_{\tilde{g}}^{4} s-t u^{2}}{\left(u+m_{\tilde{g}}^{2}\right)} \\
& \left.+\frac{1}{s} \frac{s u^{2}-u^{3}-m_{\tilde{g}}^{2} s u}{\left(u+m_{\tilde{g}}^{2}\right)}\right] \\
& +\left(4 \pi \alpha_{s}\right)^{2} 9\left[\frac{-2 m_{\tilde{g}}^{2} t-4 m_{\tilde{g}}^{4}-s t-t^{2}}{t^{2}}+\frac{m_{\tilde{g}}^{2} s-4 m_{\tilde{g}}^{4}}{t u}\right. \\
& +\frac{-m_{\tilde{g}}^{2} s-2 m_{\tilde{g}}^{2} t-s t-t^{2}}{t s}+\frac{-2 m_{\tilde{g}}^{2} u-4 m_{\tilde{g}}^{4}-s u-u^{2}}{u^{2}} \\
& \left.+\frac{-m_{\tilde{g}}^{2} s-2 m_{\tilde{g}}^{2} u-s u-u^{2}}{u s}+\frac{2 t u}{s^{2}}\right] .
\end{aligned}
$$

Here and below $s$ is the subprocess energy usually denoted by $\hat{s}$. In terms of the initial gluon momenta $q_{1}$ and $q_{2}$, and the final gluino momenta $p_{1}$ and $p_{2}, s=2 q_{1} \cdot q_{2}, t=-2 q_{1} \cdot p_{1}$ and $u=-2 q_{2} \cdot p_{1}$. In the absence of supergravitational interactions $(\kappa=0)$, the cross section for the subprocess $g g \rightarrow \tilde{g} \tilde{g}$ was first presented in Ref. [8]. Our result agrees with that of Ref. [8], if we set $\kappa=0$ in Eq. (4b).

For the process $(1 b)$, we have s-channel gluon exchange, t- and u-channel gluino exchanges, plus the contact term. The gluon gluon subprocess cross section is given by

$$
\begin{aligned}
\sigma(g g \rightarrow \tilde{g} \tilde{G})= & \frac{\alpha_{s} \kappa^{2}}{64 m_{\tilde{G}}^{2}} \frac{s-m_{\tilde{g}}^{2}}{s^{2}} \int_{-1}^{1} d z \\
& \times\left\{m_{\tilde{g}}^{2}\left[-s-\frac{2 s^{2}}{u}+\frac{2 t^{2}}{s}+4 t+2 \frac{t^{2}}{u}\right]+m_{\tilde{g}}^{4}\left[13+\frac{s}{t}+5 \frac{s}{u}+2 \frac{t}{s}+8 \frac{t}{u}\right]\right. \\
& +m_{\tilde{g}}^{6}\left[\frac{4}{s}+\frac{6}{t}+\frac{12}{u}\right] \\
& \left.+m_{\tilde{g}}^{8}\left[\frac{2}{s t}+\frac{2}{s u}+\frac{2}{t^{2}}+\frac{4}{t u}+\frac{2}{u^{2}}\right]\right\}
\end{aligned}
$$


where, in terms of the initial gluon momenta, $q_{1}$ and $q_{2}$, and the final gluino momentum $p$, we define $s=2 q_{1} \cdot q_{2}, \quad t=-2 q_{1} \cdot p, \quad u=-2 q_{2} \cdot p$. We have kept only the leading term in $1 / m_{\tilde{G}}$. For the process $(2 \mathrm{c})$, we use the coupling given by eq. (3) and obtain

$$
\begin{aligned}
& \sigma(g g \rightarrow g s+g P)=\frac{\alpha_{s}}{32} \frac{\kappa^{2}}{s} \frac{m_{\tilde{g}}^{2}}{m_{\tilde{G}}^{2}} \\
& \int_{-1}^{1} d z \frac{1}{s t u}\left\{s^{4}+2 s^{3} u+3 s^{2} u^{2}+2 s u^{3}+u^{4}\right\}
\end{aligned}
$$

where $s, t, u$ are the usual variables defined in terms of the three gluon momenta.

Next, we consider the decays of the gluinos giving rise to multijet final states. In the superlight or very light gravitino theory, the gluino decays dominantly to

$$
\tilde{g} \rightarrow g+\tilde{G} .
$$

The width is given by

$$
\Gamma(\tilde{g} \rightarrow g+\tilde{G})=\frac{\kappa^{2}}{48 \pi} \frac{m_{\tilde{g}}^{5}}{m_{\tilde{G}}^{2}} .
$$

In the usual minimal supergravity theory, the gluino decays as

$$
\tilde{g} \rightarrow q_{i} \bar{q}_{j} x, x=\text { chargino or neutralino } .
$$

In the limit of no mixing and the decay to the lightest neutralino, $\tilde{Z}_{1}$, the width is given by

$$
\Gamma\left(\tilde{g} \rightarrow q \bar{q} \tilde{Z}_{1}\right)=\frac{\alpha_{s} \alpha}{12 \pi} \frac{m_{\tilde{g}}^{5}}{m_{\tilde{q}}^{4}} \sum_{q} Q_{q}^{2}
$$

where $Q_{q}$ is the quark change, and we assume the scalar quarks to be degenerate. The relative importance of $(8)$ and (10) depends on $m_{\tilde{G}}$. In Fig. 1, we plot the branching fraction, $B(\tilde{g} \rightarrow g \tilde{G})$ vs. $m_{\tilde{G}}$ for the given gluino and squark masses, assuming that the gluino decays either to $\tilde{g} \rightarrow g \tilde{G}$ or $\tilde{g} \rightarrow q \bar{q} \tilde{Z}_{1}$ where $Z_{1}$ is the LSP. [The result is also approximately true when other 3-body decay modes are included.] As we see from Fig. 1 , for $m_{\tilde{G}}<10^{-12} \mathrm{GeV}$, the gravitino decay mode dominates. This gives rise to event topologies different from the usual minimal SG theory.

\section{Results: A Collider Bound On The Light Gravitino Mass}

The CDF collaboration, in the analysis of their Tevatron data, have used the multiple jets plus large $\mathbb{E}_{T}$ method in their search for gluino production. The minimum $\mathbb{E}_{T}$ requirement has been set fairly high, $\mathbb{E}_{T}>50 \mathrm{GeV}$, to avoid backgrounds from the SM processes, for 
example, " $Z+n$ " jets " $W+n$ " jets. CDF rules out, at $95 \% \mathrm{CL}$, a total multijet cross section greater than $1.4 \mathrm{pb}$ passing all their cuts. In our light gravitino theory, the relative importance of the production processes, (1a) vs. (1b) as well as the gluino decay modes, (7) vs. (9) depend crucially on the gravitino mass. For example, for $m_{\tilde{G}}>10^{-14} \mathrm{GeV}$, (1a) is the dominant production process and the gravitational contribution to this process through the exchange of a gravitino (the terms in (4b) that depend on $\kappa$ ) are negligible. The only effect of the gravitino is that, for $10^{-14}<m_{\tilde{G}}<10^{-12} \mathrm{GeV},(7)$ is the dominant decay mode of the gluino. In addition to the $\mathbb{E}_{T}$ cut above we used the following cuts appropriate for the CDF data:

$$
\begin{aligned}
& P_{T}>15 \mathrm{GeV} \text { for each jet } \\
& 0.1 \leq\left|\eta_{\text {jet }}\right| \leq 0.7
\end{aligned}
$$

With these cuts the CDF bound of $1.4 \mathrm{pb}$ can be used to exclude a region in the $m_{\tilde{g}}-m_{\tilde{G}}$ parameter space. To find this excluded region, we calculated the cross section, $\sigma\left(m_{\tilde{g}}, m_{\tilde{G}}\right)$ passing the signal cuts above, and plotted the contour, $\sigma\left(m_{\tilde{g}}, m_{\tilde{G}}\right)=1.4 \mathrm{pb}$ in the $m_{\tilde{g}}, m_{\tilde{G}}$ plane. The result is given by the horizontal curve in Fig. 2. For $m_{\tilde{G}}>10^{-11} \mathrm{GeV}$, the gravitational interaction is essentially negligible. Gluino-gluino production, process (1a) dominates the production cross section, and (9) dominates the gluino decay mode. We obtain $m_{\tilde{g}} \lesssim 200 \mathrm{GeV}$ as in the CDF analysis of the usual minimal SG theory. This is not shown in Fig. 2 because we are concentrating on results that bound the gravitino mass. As $m_{\tilde{G}}$ decreases to $\sim 10^{-12} \mathrm{GeV}$, the decay mode (7) becomes significant, and the curve changes due to branching ratio curve of Fig. 1. $\tilde{g} \tilde{g}$ is still the dominant production process, but the decay mode (7) now starts becoming significant. This gives rise to dominant dijet cross sections, and larger $p_{T}$ for the jets. Thus, to satisfy $\sigma \leq 1.4 \mathrm{pb}$ with the same cuts, the gluino mass must rise. For $m_{\tilde{G}}=10^{-12}$ to $10^{-14} \mathrm{GeV}$, the decay mode, $\tilde{g} \rightarrow g \tilde{G}$ is totally dominant and $\tilde{g} \tilde{g}$ production is still the dominant part of the production cross section. As a result, the curve remains flat. Below $m_{\tilde{G}}=10^{-14} \mathrm{GeV}$, the curve will go up, but this region is already excluded by our previous work [6].

Finally, we consider the exclusion region arising from the process (1c), using the couplings given by eq. (3). This process gives rise to monojets, and there is no phase space suppression. The cross section is proportional to $\left(m_{\tilde{g}} / m_{\tilde{G}}\right)^{2}$. Thus, for a given $m_{\tilde{G}}$, the CDF bound of $\sigma \leq 1.4 \mathrm{pb}$ yields an upper bound on $m_{\tilde{G}}$. The resultant exclusion region is shown by the vertical curve in Fig. 2. Combining the two excluded regions given in Fig. 2, we obtain an absolute lower bound on the light gravitino mass of about $3.0 \times 10^{-13} \mathrm{GeV}$. This is an improvement by more than a factor ten from our previous bound of $2.4 \times 10^{-14} \mathrm{GeV}$, and over a factor 100 from the previous Fayet bound of $2.3 \times 10^{-15} \mathrm{GeV}$.

\section{Conclusions}

The cuts have nearly the same effect if the final state is given by (7) as when it is given by (9). The $\mathbb{E}_{T}$ cut is almost always satisfied while the visibility of at least one of the two 
gluons, if both gluinos decay by (7), is as likely as for at least one of four quarks from (9). Thus the bound on the mass of the gluino is approximately $200 \mathrm{GeV}$ whether or not light gravitinos exist. This result is sensitive to the value of $Q^{2}$ used in the distribution functions. We use $\hat{s}$ because that gives the most conservative bound. A $Q$ value of, say, $E_{T} / 2$ gives a gluino mass bound of approximately $230 \mathrm{GeV}$. If light gravitinos do exist then the bound on their mass is given by the curves (a) and (b) in Fig. 2. For $m_{\tilde{G}}$ larger than $\sim 10^{-10}$ gravitinos are simply not produced even though they are light. Production of a gluon and a light scalar or pseudoscalar gives the bound shown by the vertical line in Fig. 2 which, together with the horizontal curve, give an absolute lower bound on the gravitino mass of $3 \cdot 0 \times 10^{-13} \mathrm{GeV}$.

\section{Acknowledgments}

S.N. wishes to thank Duane Dicus for warm hospitality and support during his sabbatical leave at The University of Texas at Austin. This work was supported in part by the U.S. Department of Energy Grants No. DE-FG013-93ER40757 and DE-FG02-94ER40852. 


\section{References}

[1] S. Park, "Search for New Phenomena in CDF," 10th Topical Workshop on ProtonAntiproton Collider Physics, eds. R. Raja and J. Yoh, AIP Press, 1995.

[2] P. Fayet, Phys. Lett. 69B, 489 (1977); J. Ellis, K. Enqvist and D.V. Nanopoulos, Phys. Lett. 147B, 99 (1984); 151B, 357 (1985); G.L. Kane and J.P. Leveille, Phys. Lett. 112B, 227 (1982); T. Bhattacharya and P. Roy, Phys. Rev. Lett. 59, 1517 (1987); Phys. Rev. D38, 2284 (1988); D.A. Dicus, S. Nandi and J. Woodside, Phys. Rev. D41, 2347 (1990); Phys. Lett. 258, 231 (1991); Phys. Rev. D43, 2951(1991).

[3] S. Ambrosanio, G.L. Kane, G.D. Kribs, S.P. Martin and S. Mrenna, Phys. Rev. Lett. 76, 3498 (1996). S. Dimopoulos, M. Dine, S. Raby and S. Thomas, Phys. Rev. Lett. 76, 3494 (1996); D.R. Stump, M. Wiest and C.P. Yuan, Phys. Rev. D54, 1936 (1996).

[4] S. Ambrosanio, G.L. Kane, G.D. Kribs, S.P. Martin and S. Mrenna, Report no. hepph/9605398; S. Dimopoulos, S. Thomas and J.D. Wells, Phys. Rev. D54, 3283 (1996); S. Dimopoulos and G.F. Giudice, Phys. Lett. B379, 105 (1996); S. Dimopoulos, G.F. Giudice and A. Pomarol, Report No. hep-ph/9607225.

[5] B.A. Barnett, CDF Collaboration, Fermilab Conf-96/039-E, Feb., 1996;

CDF Collaboration, F. Abe, et al., Phys. Rev. Lett. 76, 2006 (1996).

[6] D.A. Dicus, S. Nandi and J. Woodside, Phys. Rev. D41, 2347 (1990).

[7] E. Cremmer, S. Ferrera, L. Giradello and A. Van Proeyen, Nucl. Phys. B212, 413 (1983); R. Arnowitt, A.H. Chamsedine and P. Nath, Phys. Rev. Lett. 49, 970 (1982);

J. Bagger and E. Witten, Phys. Lett. 115B, 202 (1982); 118B, 103 (1982).

[8] P.R. Harrison and C.H. Llewellyn Smith, Nucl. Phy. B213, 223 (1983);

B223, 542 (E) (1983).

[9] P. Fayet, Phys. Lett. B175, 471 (1986). 


\section{Figure Captions}

Figure 1: Branching fraction for the gluino decay $\tilde{g} \rightarrow g \tilde{G}$. The solid line results from assuming a common squark mass of $500 \mathrm{GeV}$, the dashed line from a squark mass of $1000 \mathrm{GeV}$.

Figure 2: $m_{\tilde{g}}-m_{\tilde{G}}$ mass bound using the CDF data [Ref. 5] from the process (1a) (horizontal curves (a) for a common squark mass of $500 \mathrm{GeV}$ or (b) for a common squark mass of $1000 \mathrm{GeV}$ ) and from the process (1c) (vertical curve). The area below the horizontal curves and to the left of the vertical curve is excluded. 
This figure "fig1-1.png" is available in "png" format from: http://arxiv.org/ps/hep-ph/9611312v1 